\title{
Medicinal chemistry advances in neurodegenerative disease therapy: part 2
}

First draft submitted: 13 April 2017; Accepted for publication: 18 April 2017; Published online: 20 June 2017

Keywords: $\alpha$-synuclein $\bullet$ antiaggregating agents $\bullet$ multitarget compounds - neurodegeneration $\bullet$ neuroregeneration $\bullet$ prolyl oligopeptidase inhibitors

We are delighted to introduce the second installment of this special focus issue dedicated to medicinal chemistry advances in neurodegenerative disease therapy [1].

The WHO estimated that in April 2016 there were 47.5 million people suffering from dementia worldwide, and by 2050 this number could triple to a predicted 135.5 million [2]. Despite the efforts of a huge number of research groups around the globe, at present, all neurodegenerative diseases are incurable with only palliative treatments available.

Driven by this large and increasing need for new therapies, we have come together with renowned experts in the field to deliver the second half of our special focus issue of 'Future Medicinal Chemistry'. The issue features a range of reviews and original research covering various aspects of medicinal chemistry in neurodegenerative disease therapy.

In our first article, Simoni et al. have investigated the design and biological evaluation of hybrid compounds that target both cholinergic transmission and amyloid- $\beta$ aggregation in Alzheimer's disease, as a method to provide symptomatic relief of cholinergic potentiation and the neuroprotective prospects of antiaggregating agents [3]. This is an interesting approach and the authors present some promising compounds with activity against both of these features.

Continuing with the same aim of investigating multitarget compounds for Alzheimer's disease treatment, our second research article from Munoz-Torrero's group focuses on brain-permeable rhein-huprine hybrids [4]. These second-generation compounds have potent acetylcholinesterase or BACE-1 inhibitory activities, as well as $A \beta 42$ and tau anti-aggregating and antioxidant activities. The identified compounds, which target several crucial pathogenic factors, could be extremely useful in Alzheimer's disease treatment.

Our final research article in this special focus issue reports the development of a new potential radiotracer to be used as a probe in Alzheimer's disease. Fernández et al. investigate how an indazole-based [18F]-PETprobe can be used to detect changing levels of acetylcholinesterase in the brain [5]. Animal models demonstrated that the probe had good blood-brain barrier penetration and reasonable half-lives.

As well as featuring original research, this special focus issue contains reviews focusing on potential Alzheimer's and Parkinson's disease therapies.

The first of the review articles in this issue, from our guest editor Bolognesi and co-workers investigates the interesting possibility of targeting neuroregeneration over neurodegeneration as a strategy for Alzheimer's disease drug discovery [6]. The authors highlight that targeting the endogenous brain regenerative process using small molecules or nanoparticles may be more beneficial for patient outcomes than therapies aimed at reverting neurodegeneration.

Babkova et al. have contributed an insightful review to this special issue based

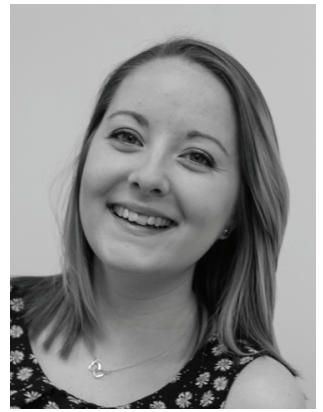

Rachel Coleby

Future Science Group - Unitec House, 2 Albert Place, London N3 1QB, UK r.coleby@future-science.com 
on naturally occurring prolyl oligopeptidase inhibitors and peptide-like inhibitors in relation to their biological effects in Alzheimer's and Parkinson's diseases [7]. Although to date, there is no prolyl oligopeptidase inhibitor available on the market, several clinical trials have been undertaken and some of them have shown promising results and interesting pharmacological profiles.

Our final article in this series focuses on the role of the $\alpha$-synuclein protein in the pathogenesis of Parkinson's disease [8]. The review from Singh et al. also covers the development of small molecules as possible modulators of $\alpha$-synuclein aggregation and their potential application in Parkinson's disease.

We hope that the articles featured in this issue of 'Future Medicinal Chemistry' provide useful insights

\section{References}

1 Bolognesi ML. Neurodegenerative drug discovery: building on the past, looking to the future. Future Med. Chem. 9(8), 707-709 (2017).

2 World Health Organization. Dementia fact sheet. www.who.int/mediacentre/factsheets/fs362/en/

3 Simoni E, Bartolini M, Abu IF et al. Multitarget drug design strategy in Alzheimer's disease: focus on cholinergic transmission and amyloid-beta aggregation. Future Med. Chem. 9(10), 953-963 (2017)

4 Pérez-Areales FJ, Betari N, Viayna A et al. Design, synthesis, and multitarget biological profiling of a second generation of anti-Alzheimer rhein-huprine hybrids. Future Med. Chem. 9(10), 965-961 (2017). into the valuable contributions being made by medicinal chemists to the advancement of neurodegenerative disease drug discovery.

\section{Acknowledgements}

Special thanks go to ML Bolognesi for serving as guest editor for our two-part special issue.

\section{Financial \& competing interests disclosure}

$\mathrm{R}$ Coleby is an employee of Future Science Group. The author has no other relevant affiliations or financial involvement with any organization or entity with a financial interest in or financial conflict with the subject matter or materials discussed in the manuscript apart from those disclosed.

No writing assistance was utilized in the production of this manuscript.

5 Fernández S, Giglio J, Reyes AL et al. 3-(Benzyloxy)-1-(5$\left[{ }^{18} \mathrm{~F}\right]$ fluoropentyl)-5-nitro-1H-indazole: a PET radiotracer to measure acetylcholinesterase in brain. Future Med. Chem. 9(10), 983-994 (2017).

6 Uliassi E, Gandini A, Perone RC, Bolognesi ML. Neuroregeneration vs neurodegeneration: towards a paradigm shift in Alzheimer's disease drug discovery. Future Med. Chem. 9(10), 995-1013 (2017).

7 Babkova K, Korabecny J, Soukup O, Nepovimova E, Jun D, Kuca K. Prolyl oligopeptidase and its role in the organism: attention to the most promising and clinically relevant inhibitors. Future Med. Chem. 9(10), 1015-1038 (2017).

8 Singh SK, Dutta A, Modi G. Alpha-synuclein aggregation modulation: an emerging approach for the treatment of Parkinson's disease. Future Med. Chem. 9(10), 1039-1053 (2017). 